\title{
Workshop: Silent Screencast Videos and Their Use When Teaching Mathematics
}

\author{
Bjarnheiður Bea Kristinsdóttir
}

Silent screencast videos are animated short videos without any text, subtitles or verbal commentary that show mathematics dynamically, focusing on one mathematical concept. The silent videos can be used in mathematics classrooms cross-culture and cross-language, giving students the assignment to add their commentary to the video.

The workshop started with an introduction explaining the background of silent screencast videos, how they are made, what they look like, and what they are used for. Next, instructions on how to add a commentary to a silent video were given and examples of commentaries made by students in Iceland were presented. In addition, participants had the opportunity to view and discuss results from experiments using the silent videos in 5th-12th grade mathematics classrooms in Estonia, Iceland, Latvia and Lithuania. After these discussions, the participants worked in groups of two to add their own commentaries to ready-made silent videos, and received guidelines on how to make their own silent videos. All workshop materials were made available on Google Drive (goo.gl/DheZ42) and some of the commentaries and silent videos created by the participants were shared in a Padlet (https://padlet. com/wall/oa1xnbbepfy9).

The activities presented in this workshop were initiated by a group of researchers and mathematics teachers from the Nordic GeoGebra Network (NGGN). NGGN was formed in 2010 to promote the use of the open-source dynamic mathematics software GeoGebra in mathematics classrooms in the Nordic and Baltic countries. The network also hosts annual conferences for teachers and education researchers. A grant from the Nordic Council of Ministers' program Nordplus made a three-year collaboration project between mathematics education researchers and mathematics teachers from the Nordic and Baltic countries possible. Each of the three years had a special theme and to address these themes, key topic groups were formed. The first

\footnotetext{
B.B. Kristinsdóttir ( $\square)$

School of Education, University of Iceland, Reykjavík, Iceland

e-mail: bjarnhek@hi.is

(C) The Author(s) 2017 
key topic group started in 2013 at the NGGN conference in Copenhagen working on the theme "Learning mathematics through screencast technology and video" and there the idea of the silent screencast videos came up. Several short silent videos showing one mathematical concept each were made, and teachers of 5th-12th grade in four different countries tested three of them in class in Autumn 2014; making their pupils write or record their own commentary to the videos. The participating teachers were surprised in a positive way: the tasks encouraged communication in the classroom and enabled teachers to "see what the pupils were thinking" (Hreinsdóttir \& Kristinsdóttir, 2016). All the tested videos were made freely available online in a GeoGebra-book (https://goo.gl/661NbX) and teachers in the different countries have continued working on new silent videos to add to the collection.

\section{Reference}

Hreinsdóttir, F., \& Kristinsdóttir, B. (2016). Using silent videos in the teaching of mathematics. In S. Ceretkova (Ed.), Staircase to even more interesting mathematics teaching (pp. 157-164). Nitra, Slovakia: Constantine the Philosophers University in Nitra.

Open Access Except where otherwise noted, this chapter is licensed under a Creative Commons Attribution 4.0 International License. To view a copy of this license, visit http://creativecommons. org/licenses/by/4.0/.

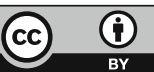

